\title{
Oxygen mass transfer to emulsions in a bubble column contactor
}

\author{
Diego Gómez-Díaz ${ }^{\mathrm{b}, *}$, Nelma Gomes ${ }^{\mathrm{a}}$, José A. Teixeira ${ }^{\mathrm{a}}$, Isabel Belo ${ }^{\mathrm{a}}$ \\ a IBB-Institute for Biotechnology and Bioengineering, Center of Biological Engineering, University of Minho, Campus de Gualtar, 4710-057 Braga, Portugal \\ ${ }^{\mathrm{b}}$ Department of Chemical Engineering, University of Santiago de Compostela, Campus Sur, E-15782 Santiago de Compostela, Spain
}

\section{A R T I C L E I N F O}

\section{Article history:}

Received 5 May 2008

Received in revised form 14 April 2009

Accepted 28 April 2009

\section{Keywords:}

Oxygen mass transfer

Emulsions

Bubble column

\begin{abstract}
A B S T R A C T
Present work deals with oxygen mass transfer in an oil-in-water biphasic medium, employed in the production of $\gamma$-decalactone by the biotransformation of methyl ricinoleate. A bubble column contactor was used to carry out the absorption experiments. The influence of operating conditions (gas flow-rate and organic phase composition) upon the gas-liquid interfacial area and mass transfer coefficient has been analysed and experimental results allow describing the mechanism of oxygen transfer from gas phase into the biphasic medium.
\end{abstract}

(c) 2009 Elsevier B.V. All rights reserved.

\section{Introduction}

Reactions involving three-phase systems are frequently found in different industrial processes. In general, the third phase (solid or liquid) dispersed in a gas-liquid system can be a reagent, substrate or a heterogeneous catalyst. Although the majority of works are focused in gas-liquid-solid systems [1-3], gas-liquid-liquid systems are gaining importance due to the increase of this type of application in bioprocess and homogeneous catalysis systems [4].

Nowadays reactors or bioreactors involving two liquid phases (organic and aqueous phases) are used as alternative of other systems because these new reactors may improve the overall process efficiency [4]. Two different fields of application have been considered for these reactors: (i) reactors to clean gaseous streams by means of the capture of pollutant gases (for instance, the presence of an organic phase could enhance the capture of volatile organic compounds due to the hydrophobic nature of these gases) [5]; (ii) bioreactors that enhance the oxygen mass transfer rate by the addition of an organic phase [6]. This is very important when the oxygen absorption is the limiting step in the global process. In these cases, an increase in the oxygen transfer rate could produce an important increase in the process productivity. Several studies have analysed the effect of different organic liquids (i.e. toluene, dodecane, heptane, etc.) [7,8] on the oxygen absorption process but different experimental results have been obtained with contradictory conclusions.

Present work uses a system formed by the emulsion of water and methyl ricinoleate (MR), stabilized by Tween 80 . This system

\footnotetext{
* Corresponding author. Fax: +34 81595012.

E-mail address: diego.gomez@usc.es (D. Gómez-Díaz).
}

has a great interest because it constitutes the biotransformation medium used in the production of $\gamma$-decalactone [9], a peach-like aroma compound of industrial interest, where the organic phase is the substrate of the process and not an inert compound added only with the aim of enhancing oxygen mass transfer. Previous studies [10] have analysed the influence of operating conditions on oxygen mass transfer and on $\gamma$-decalactone production in a bubbling stirred bioreactor. The aim of present work was to study the oxygen mass transfer mechanism in this biphasic medium, especially the influence of operating conditions to develop the absorption process and the way oxygen is transferred to the biphasic system.

\section{Materials and methods}

\subsection{Medium}

The biphasic medium used in this work consists of an aqueousliquid phase and an organic-liquid phase of MR (Stéarinerie Dubois, Boulogne, France), forming an emulsion stabilized by Tween 80 (ref. P1754, Sigma-Aldrich, Portugal), a non-ionic surfactant derived from polyethoxylated sorbitan and oleic acid, with a molecular weight of $1310 \mathrm{~g} \mathrm{~mol}^{-1}$. Different concentrations of MR (from $0 \%$ to $1.08 \%(\mathrm{v} / \mathrm{v})$ ) and Tween 80 (from $0 \%$ to $0.093 \%(\mathrm{v} / \mathrm{v})$ ) were used in experiments. Silicon anti-foaming (ref. 1.07743.0100 Merck, Portugal) was employed.

\subsection{Interfacial tension and viscosity}

Liquid-liquid interfacial tension was determined with a Krüss K-11 tensiometer using the Wilhelmy plate method. The plate employed was a commercial platinum plate supplied by Krüss. The 
platinum plate was cleaned with water and acetone and flamedried before each measurement.

The experimental set-up to carry out viscosity measurements was a capillary viscosimeter (Schott Gerate AVS 350), connected to a thermostated bath. An electronic stopwatch with an accuracy of $\pm 0.01 \mathrm{~s}$ was used for measuring efflux times.

\subsection{Oxygen mass transfer}

Oxygen mass transfer studies in the medium were carried out using a square bubble column contactor built in Perspex (polymethylmethacrylate-PMMA) with a working volume of $0.9 \mathrm{~L}$ and geometrical characteristics of $4 \mathrm{~cm}$ inside side-length and height of $65 \mathrm{~cm}$.

Air was used as gas stream in the gas-liquid contactor and it was fed at the bottom of the bubble column using a five holes sparger. The inlet gas flow-rate was measured and controlled with a mass flow controller (Alicat Scientific, USA).

For the experimental $k_{L} a$ determination, the static gassing-out technique was used [11]. Dissolved oxygen concentration was measured with a polarographic-membrane probe (12/220 T, Mettler Toledo) and monitored with a computer interface (CIODAS08JR, Computer Boards, USA) at $5 \mathrm{~s}$ intervals using the software LABtech Notebook, Datalab Solution. To estimate the probe response time $(\tau)$, the method that describes the response of the probe to a step in dissolved oxygen concentration by a first-order system was used [11]. The obtained $\tau$ value at $27^{\circ} \mathrm{C}$, of $7 \mathrm{~s}$, was used to correct $k_{L} a$ values.

Between runs, the liquid phases were de-aerated by sparging with compressed nitrogen until only minimum levels of dissolved oxygen remain ( $\approx 0 \%$ saturation). At this point, air was diffused into the bioreactor until saturation and dissolved oxygen concentration was monitored through operation time.

Other experiments of oxygen absorption in emulsions were performed in a stirred cell. In this contactor, the oxygen transfer occurs through a planar gas-liquid interface. The gas-liquid interfacial area was determined from the geometrical characteristics (internal diameter $=8 \mathrm{~cm}$ and height $=15 \mathrm{~cm}$ ) of the stirred cell. Four baffles have been placed in its internal wall to improve mixing and prevent vortex formation during stirring. The liquid phase volume used in these experiments was $250 \mathrm{~mL}$. Experimental procedure consists in the addition of different quantities of oil to the aqueous phase in the absence of Tween 80 . The absorption rate was studied with basis on the variation of oxygen concentration in the liquid phase.

\subsection{Bubble size distribution}

Bubbles diameter was measured using a photographic method based on images of the bubbles taken along the height of the column, from the bottom to the top. A Sony (DCR-PC330E) video camera was used to obtain the images. A minimum number of 50 well-defined bubbles along the bubble column were used to evaluate the size distribution of the bubbles in the liquid phase for the different gas flow-rates tested. The Image Tool v3.0 software was used to carry out the required measurements of the bubbles geometrical characteristics.

\section{Results and discussion}

\subsection{Gas-liquid interfacial area}

The use of bubble contactors involves the analysis of the gas-liquid interfacial area generated under the different experimental conditions employed in this study, in order to obtain useful information that allows the understanding of the mass transfer mechanism in this complex system based on the values of the mass

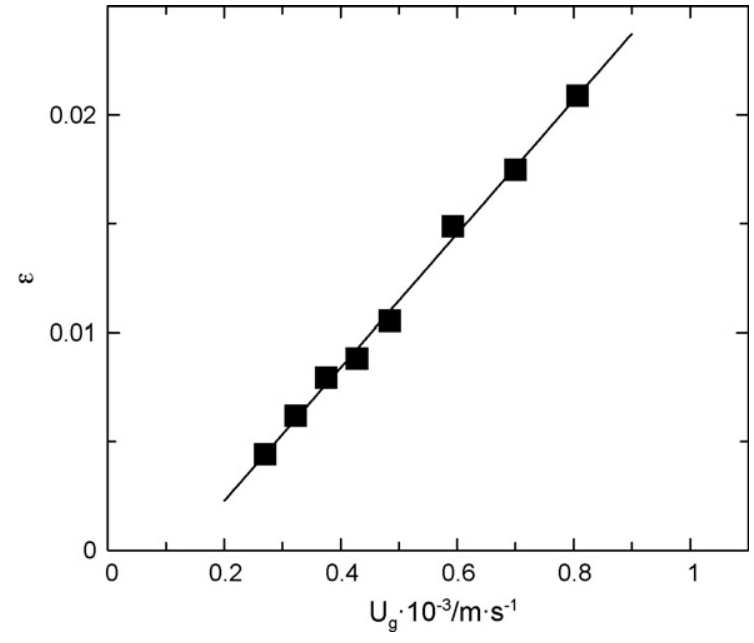

Fig. 1. Influence of surface gas velocity $\left(U_{g}\right)$ upon gas hold-up $(\varepsilon)$ for pure water.

transfer coefficient and on the influence of gas flow-rate and liquid phase composition upon this parameter. For this reason the study of gas-liquid interfacial area generated in the bubble column is the first step necessary to analyse the oxygen absorption process.

The gas hold-up is an important parameter that shows information about the flow behaviour into the bubble column. Therefore, the gas hold-up, $\varepsilon_{G}$, was calculated using the difference between the liquid height in the absence of gas in the bubble column and under the different experimental conditions of gas flow-rate fed to the contactor (Eq. (1)) [12].

$\varepsilon_{G}=\frac{\Delta V}{\Delta V+V_{L}}$

where $V_{L}$ is the ungassed liquid volume and $\Delta V$ is the volume expansion after gas dispersion, calculated from the liquid level change and the cross-sectional area. The change of volume in the bubble column was calculated based on the change observed in the liquid level and the increase of this value after gassing.

Fig. 1 shows the experimental results obtained for the gas holdup in the bubble column. This figure indicates that an increase in the gas flow-rate (or surface gas velocity) produces a clear increase in the value of the gas hold-up with a linear trend. This behaviour (for pure water) corresponds to a homogeneous regime regarding the dependency on gas flow-rate along the bubble column. Similar results for the gas hold-up have been observed for other systems employed in the present work, with surfactant and organic phase (data not shown).

The photographic method used in this work for the gas-liquid interfacial area determination was proposed in previous works $[13,14]$. The geometrical characteristics of air bubbles produced in the contactor enable to calculate the value of the gas-liquid interfacial area. Fig. 2 shows an example of the photographs taken from the bubble column. The bubbles produced in the contactor have ellipsoidal shape and for this reason, major $(E)$ and minor $(e)$ axes of the projected ellipsoid (in two dimensions) must be determined. The diameter of the equivalent sphere $\left(d_{B}\right)$ was taken as the representative bubble dimension (Eq. (2)).

$d_{B}=\sqrt[3]{E^{2} \cdot e}$

Eq. (2) allows determining the diameter distribution of a bubble present along the gas-liquid contactor. Fig. 3 shows an example of the accumulative bubble diameter distribution and the influence of surfactant concentration upon this kind of distribution. The experimental results shown in Fig. 3 allow to conclude that the presence of Tween 80 in the liquid phase produces a clear decrease in the 


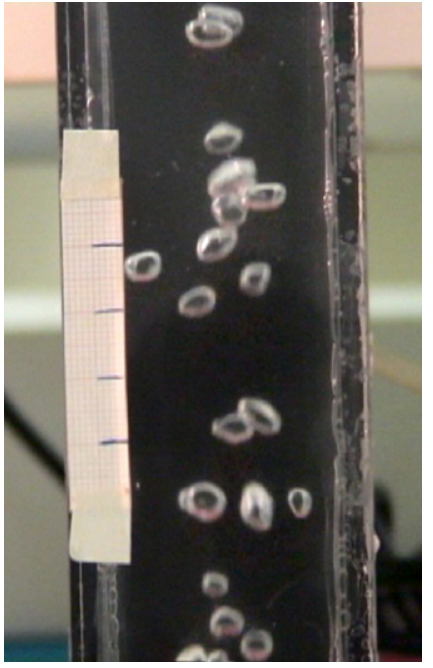

Fig. 2. Photograph at the bottom section of the bubble column using an aqueous solution of Tween 80 as liquid phase. $Q_{g}=0.5 \mathrm{~L} \mathrm{~min}^{-1}$; [ Tween 80$]=0.093 \%(\mathrm{v} / \mathrm{v})$.

bubble diameter formed in the bubble column, in agreement with Sardeing et al. [15].

The gas-liquid interfacial area can be determined by the procedure described by Painmanakul et al. [16]. The number of bubbles $\left(N_{B}\right)$ is deduced from the terminal rising bubble velocities $\left(U_{B}\right)$, the bubble formation frequency $\left(f_{B}\right)$ and the liquid height in the column $\left(H_{L}\right)$.

$N_{B}=f_{B} \cdot \frac{H_{L}}{U_{B}}$

The bubble formation frequency $\left(f_{B}\right)$ (i.e., the number of bubbles formed at the membrane orifice per unit time) is determined using Eq. (4).

$f_{B}=\frac{Q_{G}}{V_{B}}$

where $V_{B}$ is the average detached bubble volume and $Q_{G}$ is the gas flow-rate fed to the bubble column.

Due to the image treatment system, the terminal rising bubble velocities $\left(U_{B}\right)$ can be estimated for the most frequent bubble

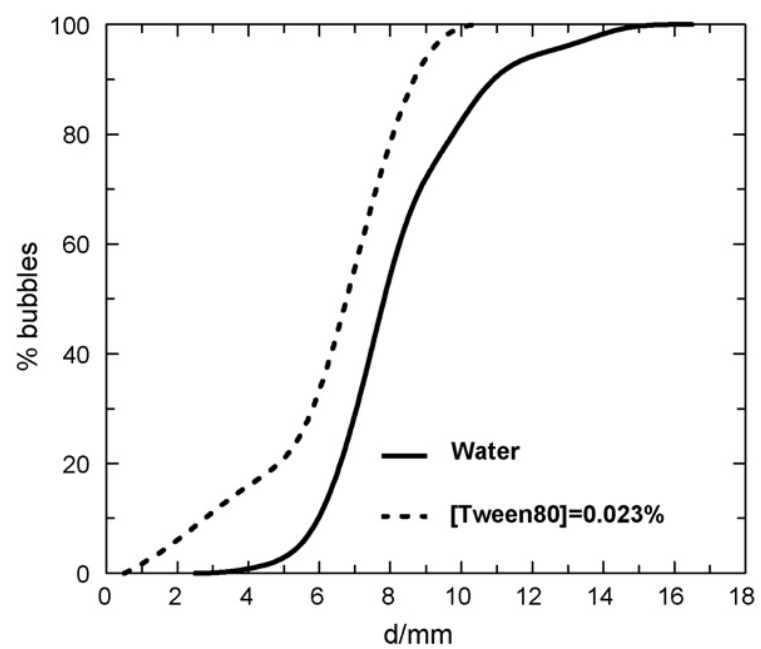

Fig. 3. Influence of Tween 80 concentration in the liquid phase upon the bubble size. $Q_{g}=0.5 \mathrm{Lmin}^{-1}$

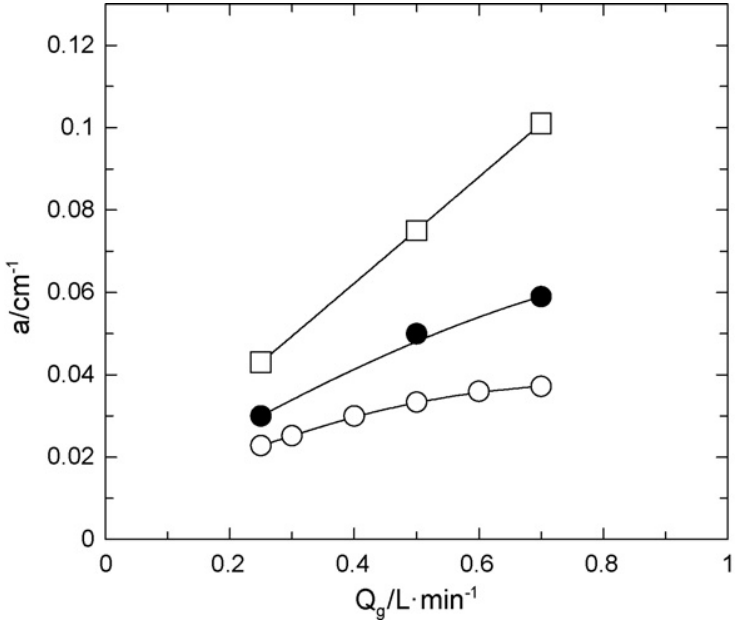

Fig. 4. Influence of gas flow-rate $\left(Q_{g}\right)$ and Tween 80 concentration in the liquid phase upon gas-liquid interfacial area $(a)$. $(\bigcirc)$ [Tween 80$]=0 \%$; $(\bullet)$ [Tween 80$]=0.023 \%$; ( $\square$ ) [Tween 80$]=0.093 \%$.

diameter:

$U_{B}=\frac{\Delta y}{\Delta t}$

where $\Delta y$ is the variation through the column height of the bubble column and $\Delta t$ is the variation of time.

The gas-liquid interfacial area is determined as the ratio between the bubble surfaces $\left(S_{B}\right)$ and the total volume in reactor $\left(V_{T}\right)$ by means of Eq. (6).

$a=N_{B} \cdot \frac{S_{B}}{V_{T}}$

Depending on the bubble shape (spherical and ellipsoidal) the bubble surface must be determined using different equations (Eqs. (7) and (8)) [16].

Spherical shape : $S_{B}=\pi \cdot D_{B}^{2}$

Ellipsoidal shape : $S_{B}=2 \cdot \pi \cdot\left[\frac{E^{2}}{4}+\left(\frac{E^{2}}{4} \cdot \frac{1}{2 \cdot \alpha} \cdot \ln \left(\frac{1+\alpha}{1-\alpha}\right)\right)\right]$

where $\alpha$ is the ratio between the major $(E)$ and minor $(e)$ axis of the bubble. (9).

On the other hand the total volume is determined employing Eq.

$V_{T}=A \cdot H_{L}+N_{B} \cdot V_{B}$

where $A$ is the bubble column cross-sectional area.

This experimental methodology allows the determination of gas-liquid interfacial area under the different experimental conditions (liquid phase composition and gas flow-rate) assayed in this work.

Experimental results shown in Fig. 4 indicate the influence of liquid phase composition and gas flow-rate upon the specific interfacial area generated in the bubble column. When gas flow-rate increases, the interfacial area also increases due to the previously observed behaviour caused by this variable upon the gas hold-up (Fig. 1). The gas hold-up increases with the gas flow-rate because a higher number of bubbles are generated in the sparger, thus increasing the interfacial area. Moreover, since a homogeneous regime is maintained, an increase in the gas flow-rate also increases the bubble size due to low grade of coalescence, but with lower effect upon interfacial area than gas hold-up.

When pure water was employed as liquid phase in the bubble contactor at high values of gas flow-rate, that increase was not 


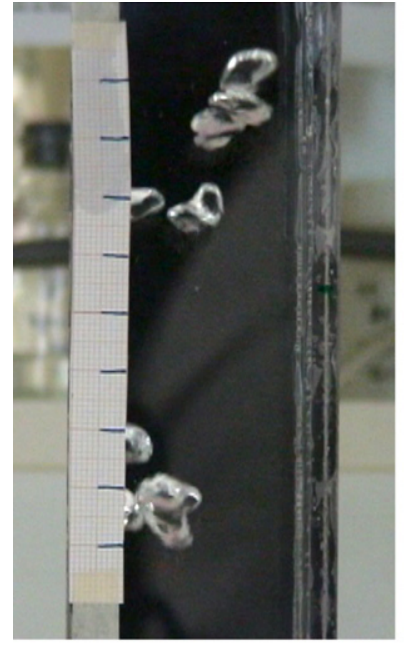

Pure water

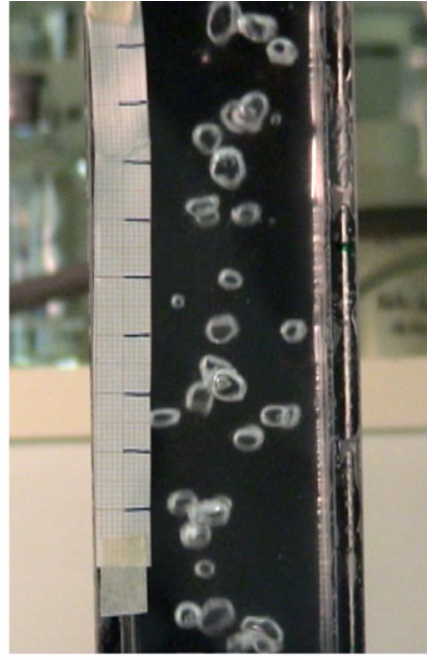

[Tween 80] $=0.093 \%$
Fig. 5. Photographs from middle section of the bubble column. $Q_{g}=0.5 \mathrm{~L} \mathrm{~min}^{-1}$.

observed because at high values of gas flow-rate there is a great number of bubbles that collide producing coalescence and then, the increase in the bubbles diameter. This increase in the bubbles size has a negative effect upon the gas-liquid interfacial area and reduces the positive effect caused by the gas hold-up. Then for pure water the effect caused by coalescence upon gas-liquid interfacial area is higher than the influence caused by gas hold-up.

The presence of surfactant in the liquid phase produces a clear increase in the value of the specific interfacial area (Fig. 4) and the increase regards the corresponding value for pure water is higher when the gas flow-rate increases. This behaviour is due to different reasons. One of them is the clear decrease in the liquid phase surface tension when the surfactant concentration increases [17] as it causes the formation of bubbles with minor size, increasing the interfacial area [18]. Also, the presence of surfactant molecules in the liquid phase produces a decrease in the bubbles effective collisions and then the bubbles small size remains constant along the bubble column [19].

Photographs in Fig. 5 confirm that the presence of Tween 80 in the liquid phase produces a decrease in the bubbles size, implying an increase in the gas-liquid interfacial area.

After study the influence of Tween 80 upon the bubbles diameter, gas hold-up and gas-liquid interfacial area, the effect of organic phase addition to the aqueous solutions was also analysed. For this study, different concentrations of methyl ricinoleate (from $0 \%$ to $0.54 \%(\mathrm{v} / \mathrm{v})$ ) have been added. The operation conditions regarding the gas flow-rate were kept in the same range of values as used in the experiments depicted above. Surfactant concentration remained constant at its higher value $(0.093 \%(\mathrm{v} / \mathrm{v}))$ in order to maintain the stability of the emulsion. The concentration of organic phase was modified to analyse its influence upon the interfacial area. Fig. 6 shows the influence of organic phase presence upon the bubbles size. As previously mentioned, the addition of Tween 80 to pure water produces a clear decrease in the bubbles size, however when oil is added to the aqueous phase, a continuous increase in the bubbles diameter is observed and, for the highest oil concentration, the bubbles diameter are higher than the corresponding ones for pure water.

Using the same procedure than the previously employed for aqueous solutions of Tween 80 , the gas-liquid interfacial area was calculated for the oil-in-water emulsions. The experimental results obtained for these systems are presented in Fig. 7 that show a decrease in the specific interfacial area value when the oil concen-

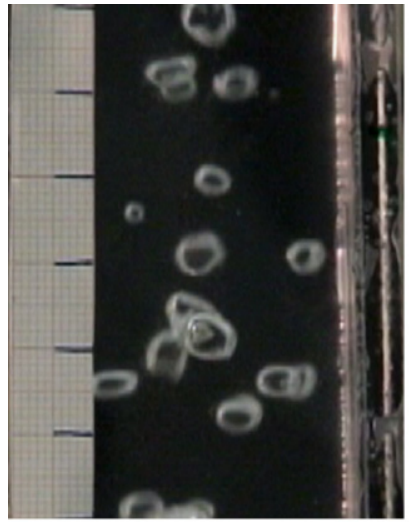

[Tween 80] $=0.093 \%$ $[\mathrm{MR}]=0 \%$

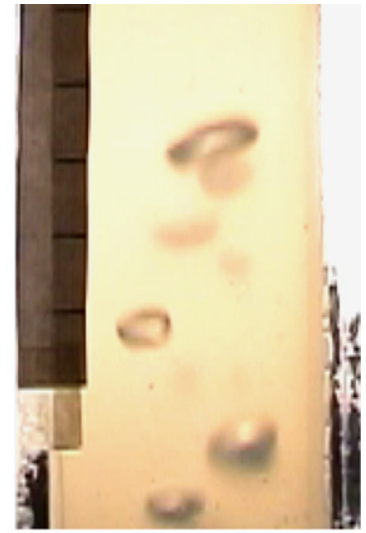

[Tween 80] $=0.093 \%$ $[\mathrm{MR}]=0.54 \%$

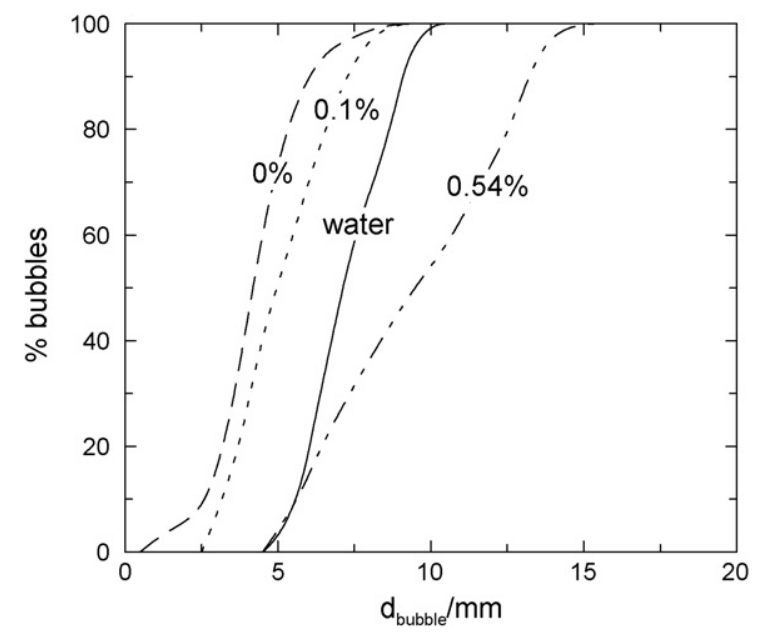

Fig. 6. Influence of methyl ricinoleate concentration upon the bubbles size distribution at middle section for water and emulsions. $Q_{g}=0.5 \mathrm{~L} \mathrm{~min}^{-1}$.

tration increases in the liquid phase. This effect is negligible for the lowest gas flow-rate analysed but when it increases, the presence of the organic phase has an important contribute on the values of interfacial area.

This behaviour is assigned to a change in the surfactant concentration at gas-liquid interface because when an organic phase is added to aqueous solutions with Tween 80 , a new distribution of

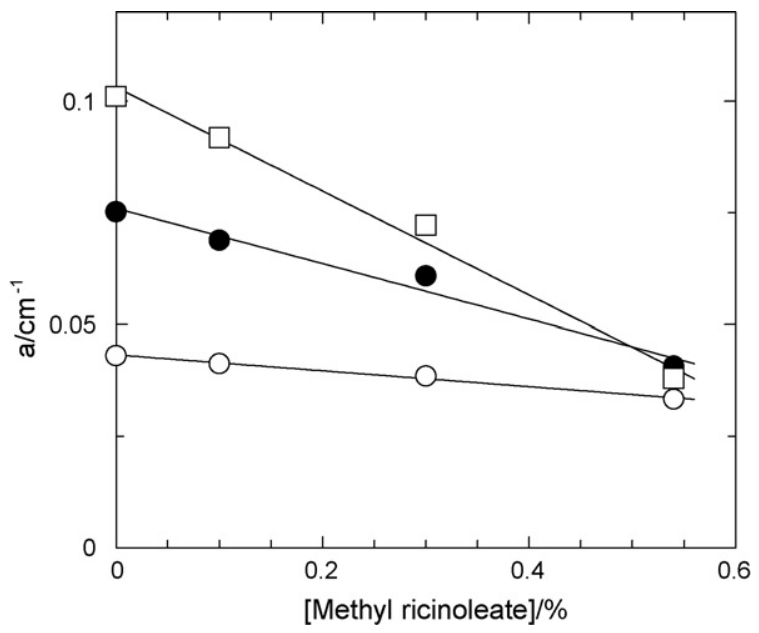

Fig. 7. Influence of methyl ricinoleate concentration on the interfacial area in the bubble column. $(\bigcirc) Q_{g}=0.25 \mathrm{~L} \mathrm{~min}^{-1} ;(\bullet) Q_{g}=0.5 \mathrm{~L} \mathrm{~min}^{-1}$; $(\square) Q_{g}=0.7 \mathrm{~L} \mathrm{~min}^{-1}$. 


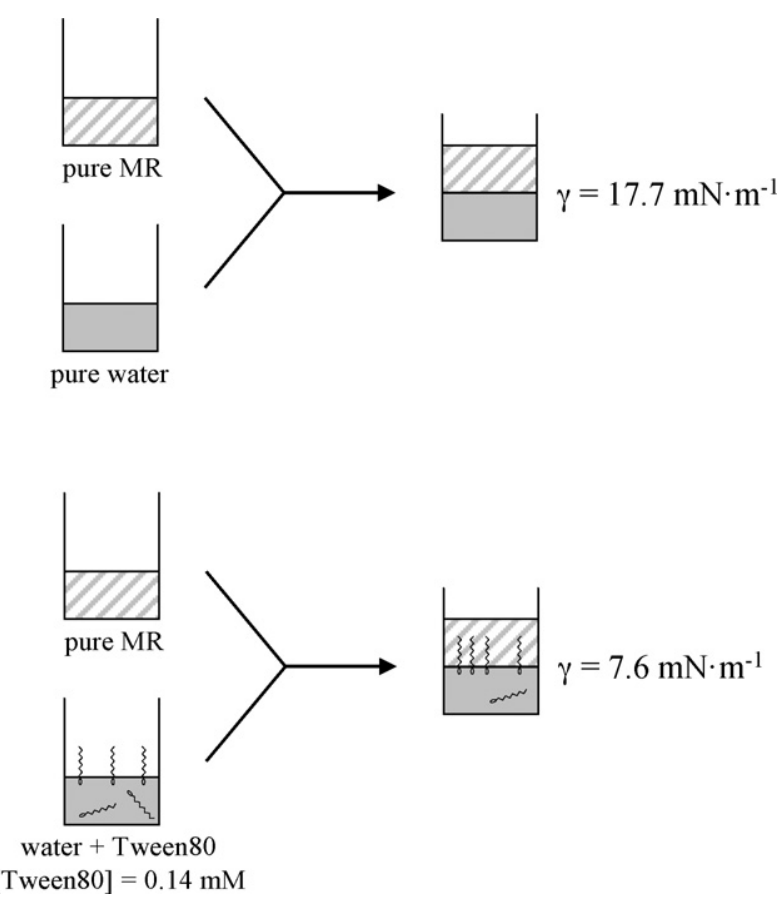

Fig. 8. Effect of Tween 80 upon the interfacial tension in water-methyl ricinoleate system.

surfactant between interface and bulk phases is originated. Interfacial tension measurements between water and methyl ricinoleate allowed confirming this assumption, because a decrease in the value of interfacial tension was observed when Tween 80 was added to the liquid-liquid biphasic system (Fig. 8). This distribution affects the value of the liquid phase surface tension and consequently bubbles produced under these conditions have higher diameters than the ones formed in the absence of organic phase.

Also, the presence of the surfactant in the aqueous phase reduced the coalescence process but in this case, the concentration of surfactant in the gas-water interface decreases and consequently the reduction in coalescence is minor and the bubbles increase their diameter in the ascension along the bubble column.

\subsection{Gas-liquid mass transfer}

Semi-continuous regime has been employed in the present work and Eq. (10) was used to determine the volumetric mass transfer coefficient obtained, using oxygen concentration data along the time.

$\frac{d C}{d t}=k_{L} a \cdot\left(C^{*}-C\right)$

where $k_{L} a$ is the volumetric mass transfer coefficient, and $C^{*}$ and $C$ are the solubility and dissolved oxygen concentrations, respectively. To use this equation, it is assumed that the mixture is homogeneous within the reactor and the volumetric mass transfer coefficient was determined taking into account the constant time of the oxygen probe.

Liquid side mass transfer coefficient has been calculated as the quotient between volumetric mass transfer coefficient and the specific gas-liquid interfacial area determined previously (Section 3.1).

The influence of surfactant concentration upon the value of the mass transfer coefficient is shown in Fig. 9. An addition of Tween 80 to the liquid phase produces an important decrease in the mass transfer coefficient due to the presence of surfactant molecules at the gas-liquid interface [12]. The presence of surfactant inhibits the mass transfer because its accumulation at gas-liquid interface

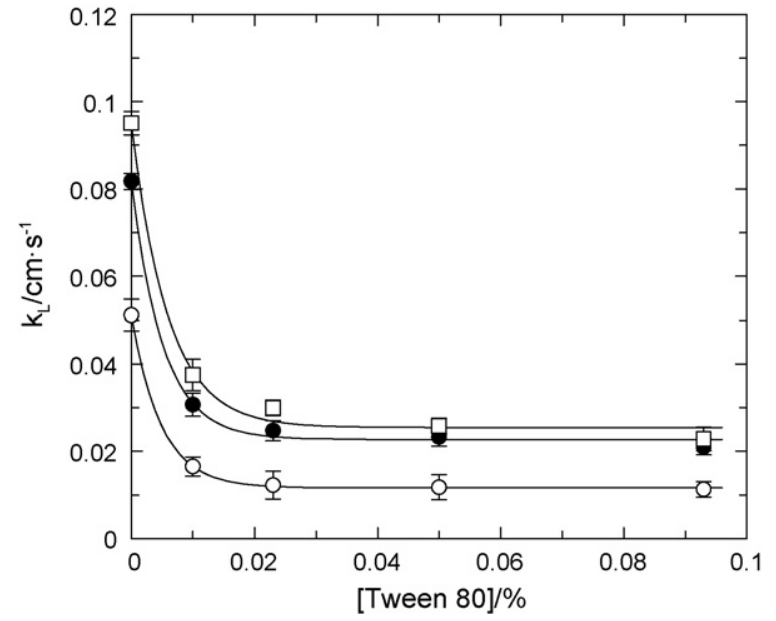

Fig. 9. Influence of surfactant concentration upon the liquid side mass transfer coefficient. ( $\bigcirc) Q_{g}=0.25 \mathrm{~L} \mathrm{~min}^{-1} ;(\bullet) Q_{g}=0.5 \mathrm{~L} \mathrm{~min}^{-1}$; $(\square) Q_{g}=0.7 \mathrm{~L} \mathrm{~min}^{-1}$.

causes a decrease in the liquid surface elements renewal (turbulence near the interface) and it entails that oxygen concentration in the liquid phase near to the gas-liquid interface is higher than the oxygen concentration in the bulk of the liquid phase. Therefore, the driving force of the absorption process decreases producing the same decrease in the absorption rate.

A similar behaviour has been found by other researchers when they analysed the influence of surfactant concentration in the liquid phase in an absorption process [16]. An important decrease in the mass transfer coefficient at low surfactant concentrations is observed and then a slightly decrease is produced when the surfactant concentration increases in the liquid phase. This slightly decrease in the higher concentration range, is related with the critical micelle concentration of the surfactant in the solvent $\left(\mathrm{CMC}_{\text {Tween } 80}=0.012 \mathrm{mM}\right)[20]$

Fig. 9 shows also that an increase in the air flow-rate leads to an increment in the mass transfer coefficient. This effect is due to an increase in the turbulence caused by the bubbles ascension along the bubble column.

The effect of the organic phase on the mass transfer coefficient in this bubble contactor has also been analysed and the experimental results are shown in Fig. 10. This figure shows that the presence of methyl ricinoleate in the liquid phase produces an increase in the mass transfer coefficient that is more pronounced at high values

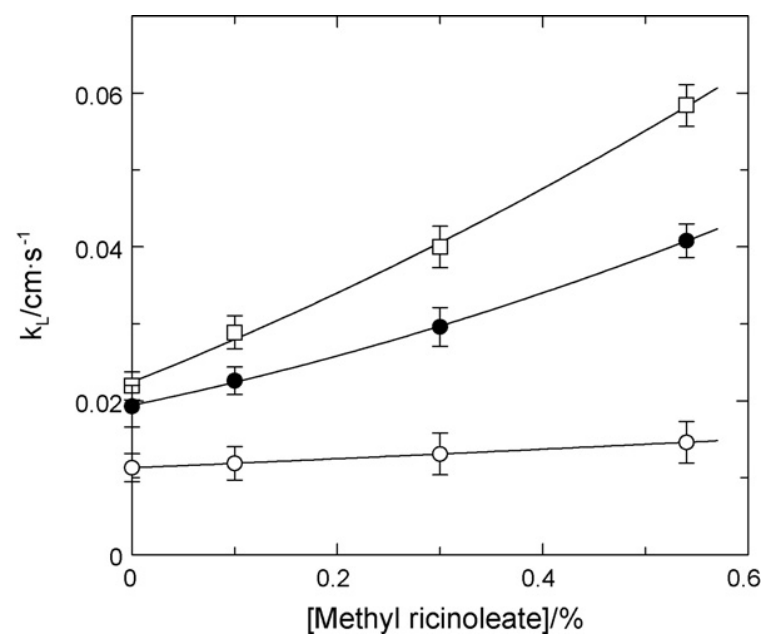

Fig. 10. Influence of organic phase concentration upon the mass transfer coefficient (○) $Q_{g}=0.25 \mathrm{Lmin}^{-1} ;(\bullet) Q_{g}=0.5 \mathrm{Lmin}^{-1} ;(\square) Q_{g}=0.7 \mathrm{~L} \mathrm{~min}^{-1}$. 


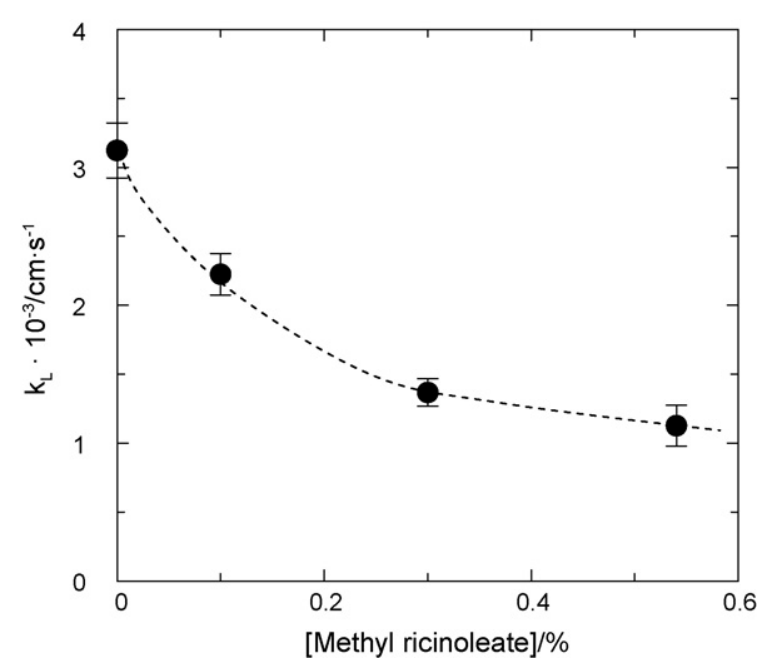

Fig. 11. Effect of organic phase on volumetric mass transfer coefficient, in a flat stirred vessel. $Q_{g}=0.333 \mathrm{~L} \mathrm{~min}^{-1}$.

of gas flow-rate. These results are in agreement with a previous study carried out in a stirred tank contactor with this same system, where an increase in the volumetric mass transfer coefficient in the presence of organic phase was observed [10].

The explanation used for the influence on interfacial area can also be used to justify the behaviour observed regarding the mass transfer coefficient. Therefore, the presence of methyl ricinoleate in the liquid phase produces a new distribution of the surfactant molecules and then a decrease in Tween 80 concentration in the gas-liquid interface must be originated. The presence of surfactant molecules produces a negative effect upon the mass transfer coefficient (Fig. 9) due to a reduction of liquid renewal at interface. Then, the presence of methyl ricinoleate causes an increase in the driving force and it improves the oxygen transfer to the bulk of the liquid phase. This new situation caused by the presence of organic phase is positive for mass transfer.

Other authors have studied the absorption process in biphasic systems and they have observed a maximum in the value of the oxygen mass transfer rate, but for higher organic phase concentrations [21].

The influence of gas flow-rate upon the mass transfer coefficient value for the emulsion system is similar to previous results for aqueous solutions of Tween 80 , occurring an increase in its values when the gas flow-rate also increases. This is caused by the enhancement of turbulence in the liquid phase and at gas-liquid interface.

Several studies $[8,22]$ show that the influence of an organic phase on absorption processes can be due to the higher absorption capacity of the organic liquid, enhancing the mass transfer. For this reason, additional experimental work has been developed in a flat stirred vessel. Results obtained (Fig. 11) show that an increase in the presence of the organic phase over the water interface produces a decrease in the volumetric mass transfer coefficient (the interfacial area remains constant). This behaviour is due to the higher viscosity of the organic phase $\left(v_{\mathrm{MR}}=68 \mathrm{~mm}^{2} \mathrm{~s}^{-1}\right)$ when compared with water $\left(v_{\text {water }}=0.9 \mathrm{~mm}^{2} \mathrm{~s}^{-1}\right)$, producing a negative effect upon the mass transfer rate since it reduces the value of gas diffusivity in the liquid phase. Therefore, we consider that the positive effect on the mass transfer coefficient caused by the presence of methyl ricinoleate in this contactor (Fig. 10) is due to the reduction in Tween 80 concentration at gas-liquid interface.

\section{Conclusions}

Present work analysed the absorption process of oxygen from an air stream towards a liquid heterogeneous phase composed of an aqueous and an organic phase, forming an emulsion, in a bubbling contactor. The complex nature of this kind of liquid phase implies the knowledge of the influence of each one of the components on the gas-liquid mass transfer process.

Hereby, the presence of the surface active substance used to stabilize the emulsion has a significant effect both on the interfacial area generated in the bubble column and on the mass transfer coefficient due to the accumulation trend of this kind of compound at the gas-liquid interface. The presence of the organic phase has a positive effect on the oxygen mass transfer rate to the liquid phase, but on the other hand, it produces a very clear decrease of the interfacial area, which has a negative effect on the global absorption process.

\section{Acknowledgements}

The authors acknowledge the financial support provided by CRUP and Ministerio de Ciencia e Innovación through the bilateral cooperation E-101/08 and HP2007-0059, and by Fundação para a Ciência e Tecnologia through the grant SFRH/BD/28039/2006.

\section{References}

[1] P.C. Mena, M.N. Pons, J.A. Teixeira, F.A. Rocha, Using image analysis in the study of multiphase gas absorption, Chem. Eng. Sci. 60 (2005) 51445150.

[2] X. Zhang, S. Schubert, M. Gruenewald, D.W. Agar, Studies on the kinetics of carbon dioxide absorption with immobilised amines (IA), Chem. Eng. J. 107 (2005) 97-102.

[3] M.V. Dagaonkar, H.J. Heeres, A.A.C.M. Beenackers, V.G. Pangarkar, The application of fine $\mathrm{TiO}_{2}$ particles for enhanced gas absorption, Chem. Eng. J. 92 (2003) 151-159.

[4] E. Dumont, H. Delmas, Mass transfer enhancement of gas absorption in oil-in-water systems: a review, Chem. Eng. Process. 42 (2003) 419438.

[5] E. Dumont, Y. Andrès, P. Le Cloirec, Mass transfer coefficients of styrene and oxygen into silicone oil emulsions in a bubble reactor, Chem. Eng. Sci. 61 (2006) 5612-5619.

[6] E. Dumont, Y. Andres, P. Le Cloirec, Effect of organic solvents on oxygen mass transfer in multiphase systems: application to bioreactors in environmental protection, Biochem. Eng. J. 30 (2006) 245-252.

[7] T.L. da Silva, V. Calado, N. Silva, R.L. Mendes, S.S. Alves, J.M.T. Vasconcelos, A. Reis, Effects of hydrocarbon additions on gas-liquid mass transfer coefficients in biphasic bioreactors, Biotech. Bioproc. Eng. 11 (2006) 245250.

[8] A.H.G. Cents, D.W.F. Brilman, G.F. Versteeg, Gas absorption in an agitated gas-liquid-liquid system, Chem. Eng. Sci. 56 (2001) 1075-1083.

[9] M. Aguedo, N. Gomes, E. Escamilla-Garcia, Y. Waché, M. Mota, J.A. Teixeira, I. Belo, Decalactone production by Yarrowia lipolytica under increased $\mathrm{O}_{2}$ transfer rates, Biotechnol. Lett. 27 (2005) 1617-1621.

[10] N. Gomes, M. Aguedo, J.A. Teixeira, I. Belo, Oxygen mass transfer in a biphasic medium: Influence on the biotransformation of methyl ricinoleate into $\gamma$-decalactone by the yeast Yarrowia lipolytica, Biochem. Eng. J. 35 (2007) 380386.

[11] A. Tribe, C.L. Briens, A. Margaritis, Determination of the volumetric mass transfer coefficient $\left(k_{\mathrm{L}} a\right)$ using the dynamic "gas out-gas in" method: analysis of errors caused by dissolved oxygen probes, Biotechnol. Bioeng. 46 (1995) 388-392.

[12] J.M.T. Vasconcelos, J.M.I. Rodrigues, S.C.P. Orvalho, S.S. Alves, R.L. Mendes, A. Reis, Effect of contaminants on mass transfer coefficients in bubble column and airlift contactors, Chem. Eng. Sci. 58 (2003) 1431-1440.

[13] A.A. Sotiriadis, R.B. Thorpe, J.M. Smith, Bubble size and mass transfer characteristics of sparged downwards two-phase flow, Chem. Eng. Sci. 60 (2005) 5917-5929.

[14] A. Couvert, M. Roustan, P. Chatellier, Two-phase hydrodynamic study of a rectangular airlift loop reactor with an internal baffle, Chem. Eng. Sci. 54 (1999) 5245-5252.

[15] R. Sardeing, P. Painmanakul, G. Hébrard, Effect of surfactants on liquid-side mass transfer coefficients in gas-liquid systems: a first step to modelling, Chem. Eng. Sci. 61 (2006) 6249-6260.

[16] P. Painmanakul, K. Loubière, G. Hébrard, M. Mietton-Peuchot, M. Roustan, Effect of surfactants on liquid-side mass transfer coefficients, Chem. Eng. Sci. 60 (2005) 6480-6491.

[17] Y.T. Shah, B.G. Kelkar, S.P. Godbole, W.-D. Deckwer, Design parameters estimation for bubble column reactors, AIChE J. 28 (1982) 353-379.

[18] E.A. Vogler, K.B.Spencer, D.B. Montgomery, L.M. Lander, W.J. Brittain, Design and operational characteristics of a robotic Wilhelmy balance, Langmuir 9 (1993) 2470-2477. 
[19] J. Lee, S.E. Kentish, M. Ashokkumar, The effect of surface-active solutes on bubble coalescence in the presence of ultrasound, J. Phys. Chem. B 109 (2005) 5095-5099.

[20] M.E. Haque, A.R. Das, S.P. Moulik, Mixed micelles of sodium deoxycholate and polyoxyethylene sorbitan monooleate (Tween 80), J. Colloid Interface Sci. 217 (1999) 1-7.
[21] D.R. Nielsen, A.J. Daugulis, P.J. McLellan, A novel method of simulating oxygen mass transfer in two-phase partitioning bioreactors, Biotechnol. Bioeng. 83 (2003) 735-742.

[22] A. Lekhal, R.V. Chaudhari, A.M. Wilhelm, H. Delmas, Gas-liquid mass transfer in gas-liquid-liquid dispersions, Chem. Eng. Sci. 52 (1997) 4069-4077. 